\title{
Off-label-use in der Onkologie: keine maximale, sondern optimale Medizin
}

\section{Jürg Nadiga, Thomas Kroner ${ }^{b}$, Jürg Zollikoferc}

a Dr. med., Facharzt für Medizinische Onkologie und Innere Medizin FMH, Präsident der Schweizerischen Gesellschaft für Medizinische Onkologie

b Dr. med., Facharzt für Medizinische Onkologie und Innere Medizin FMH, Leiter der Paritätischen Arbeitsgruppe SGV-SGMO

c Dr. med., Facharzt für Allgemeinmedizin FMH, Präsident der Schweizerischen Gesellschaft der Vertrauensärzte

\section{Paritätische Arbeitsgruppe SGV-SGMO}

Um die Entscheide im Off-label-use zu vereinheitlichen, beraten die Medizinischen Onkologen die Vertrauensärzte in einer paritätischen Arbeitsgruppe der Schweizerischen Gesellschaft der Vertrauens- und Versicherungsärzte (SGV) und der Schweizerischen Gesellschaft für Medizinische Onkologie (SGMO) [1]. Ein aktueller Bundesgerichtsentscheid (BGE) [2] wird die Arbeit dieser Kommission beeinflussen. Im Urteil vom 23.11.2010 gibt die sozialrechtliche Abteilung des Bundesgerichts (BG) einer Krankenversicherung recht, die sich geweigert hat, die hohen Kosten für eine Myozymbehandlung bei der adulten Form des Morbus Pompe zu übernehmen. Obwohl es sich um eine Orphan Disease mit einer Orphan Drug handelt, beeinflusst der BGE auch die Beurteilung von Offlabel-Indikationen.

\section{Bestehende Regeln bestätigt und präzisiert}

In seiner Argumentation wiederholt das BG die heute geltenden Regeln im Off-label-Bereich:

Die Kosten für eine Off-label-Behandlung sind von der Obligatorischen Krankenpflegeversicherung (OKP) zu übernehmen, wenn die Krankheit tödlich verläuft oder schwere und chronische gesundheit-

\section{«Kriterium für die Beschaffung und die Verteilung staatlicher Mittel sei die Verteilungsgerechtigkeit.»}

1 Nadig J. Off Label Use: Pragmatische Lösung eines komplexen Problems. Schweizer Krebsbulletin 2010;30:271.

2 BGE 9C 334.201

Korrespondenz: Dr. med. Jürg Nadig MAE Bannhaldenstrasse 7 CH-8180 Bülach Tel. 0448627300 Fax 0448627300 juerg.nadig@hin.ch liche Probleme nach sich zieht und therapeutische Alternativen fehlen. Das Arzneimittel muss einen hohen therapeutischen (kurativen oder palliativen) Nutzen haben.

\section{Therapeutischer Nutzen}

Das Bundesgericht präzisiert in seinem Urteil einzelne Begriffe der BAG-Verordnung: Im Off-labelBereich könne nicht jeder therapeutische Nutzen genügen, da sonst die Einzelfallbeurteilung an die Stelle des gesetzlichen Listensystems trete und dieses unterwandere. Es müsse vermieden werden, dass durch eine extensive Praxis der ordentliche Weg der Listenaufnahme durch Einzelfallbeurteilung ersetzt und dadurch die mit der Spezialitätenliste verbundene Wirtschaftlichkeitskontrolle durch die Preisfest- setzungsbehörde umgangen wird. Der hohe therapeutische Nutzen wird als ein allgemeiner Wirksamkeitsnachweis umschrieben. Die Wirkung am Einzelfall allein könne nicht genügen. Um ein Medikament im Off-label-Bereich zu Lasten der OKP zu übernehmen, braucht es keine Heilung und keine hohe Remissionsrate, sondern die Wirksamkeit muss in einer Studie nachgewiesen sein, bevor es im Einzelfall übernommen werden kann. Eine Wunderheilung kann also nicht zu Lasten der OKP übernommen werden.

Das Bundesgericht weist ferner darauf hin, dass die Frage nach dem hohen therapeutischen Nutzen nicht mit einem kategorialen Ja oder Nein beantwortet werden könne: Die Frage nach dem hohen therapeutischen Nutzen sei graduell und in Relation zu den Behandlungskosten zu beurteilen.

\section{Prüfungskriterien}

Bei der Prüfung der Wirksamkeit einer Off-label-Indikation sind nicht die gleich strengen Kriterien an den Wirksamkeitsnachweis gefordert, wie bei einer generellen Aufnahme in die Spezialitätenliste. Die Studiendaten sollten lediglich rechtfertigen, im Einzelfall vom Listenerfordernis abzuweichen. Wirksamkeit und Zweckmässigkeit müssen in einer wissenschaftlichen Studie nachgewiesen sein. Die minimalen Voraussetzungen für einen Wirksamkeitsnachweis sollen die gleichen sein wie bei der befristeten Bewilligung nicht zugelassener Arzneimittel: Zwischenergebnisse von Studien weisen darauf hin, dass ein grosser therapeutischer Nutzen zu erwarten ist. Ohne wissenschaftliche Daten, die die Wirksamkeit beschreiben, kann kein Medikament off label übernommen werden.

\section{Wirtschaftlichkeitsprüfung im Off-label- Bereich}

Auch im Off-label-Bereich ist die Wirtschaftlichkeit einer Behandlung zu prüfen. Anhand verschiedener Gerichtsentscheide tastet sich das Bundesgericht an einen Kostenrahmen heran, der von der OKP zu übernehmen ist. Wo staatlich administrierte Güter nicht unbegrenzt zur Verfügung stehen, ist eine möglichst rechtsgleiche Verteilung anzustreben; es soll vermieden werden, dass die einen alles oder sehr viel und die anderen nichts oder fast nichts erhalten. Es gehe deshalb nicht an, dass für die Heilung eines einzelnen Kranken jeder Preis bezahlt werde, weil sonst die Mit- 
tel an einem anderen Ort fehlten. Kriterium für die Beschaffung und die Verteilung staatlicher Mittel sei die Verteilungsgerechtigkeit. Rechtsgleichheit setze Verallgemeinerungsfähigkeit voraus. Verallgemeinerungsfähig sei aber nur, was allen, die sich in der gleichen Situation befinden, in gleicher Weise angeboten werden kann. Ohne besondere Rechtfertigung sei es mit der Rechtsgleichheit und der Gleichwertigkeit aller Menschen nicht vereinbar, einzelnen Versicherten Leistungen zu erbringen, die anderen Versicherten in gleicher Lage nicht erbracht werden können.

Damit verlangt die Beurteilung einer Off-labelIndikation auch für die Wirtschaftlichkeit einheitliche Kriterien, damit nicht die Zugehörigkeit zu einer Versicherung oder die Postleitzahl über den Zugang zu einer Behandlung entscheidet.

\section{Obergrenze für eine medikamentöse Therapie} Gemäss diesem Prinzip berechnet das Bundesgericht die Therapiejahreskosten. Beim Morbus Pompe begrenzt die Atemnot wegen einer Schwäche der Atem- halb nicht zu Lasten der OKP übernommen werden. Monatliche Medikamentenkosten von 7000 Franken beim fortgeschrittenen Kolonkarzinom gelten noch als wirtschaftlich, ebenso Spitexkosten von 100000 Franken pro Jahr, auch wenn die gleiche Pflege im Pflegeheim dreieinhalb Mal günstiger erbracht werden kann. In beiden Fällen handelt es sich nicht um Kosten pro gewonnenes Lebensjahr, sondern um Behandlungskosten pro Jahr.

\section{Was heisst das für die Arbeitsgruppe der Vertrauensärzte und Onkologen?}

Als erstes ist aufgrund von Studien zu prüfen, ob die Wirksamkeit ein Abweichen von der Listenzulassung rechtfertigt. Ferner müssen auch die Kosten einer Therapie berücksichtigt werden. Kann die Wirksamkeit gemäss den Kriterien des BAG bejaht werden, wird im Preissegment bis 8000 Franken Monatskosten bei metastasierenden Krankheiten die Kostengutsprache für die Zeit erteilt, die nötig ist, um das Ansprechen beim Patienten zu beurteilen. Die Ansprech-
* www.vertrauensaerzte.ch/ expertcom/oluoncology/ methods.html

\section{Der Medikamentenpreis könnte so gesenkt werden, dass die adjuvante Behandlung nicht mehr Kosten verursacht als die Behandlung mit dem gleichen Medikament in der palliativen Situation.}

muskulatur die Mobilität der Kranken. In der Schweiz gebe es 2,5\% der Einwohner, die wegen einer schweren COPD gleich eingeschränkt seien wie die Patientin mit Morbus Pompe. Müsste ihnen allen eine gleich teure Therapie zugänglich gemacht werden, wäre allein für diese fiktive Behandlung 1,5 mal das Gesamtbudget des öffentlichen Gesundheitswesens aufzuwenden. Damit sei die Behandlung nicht verallgemeinerungsfähig. So hohe Kosten könnten des-

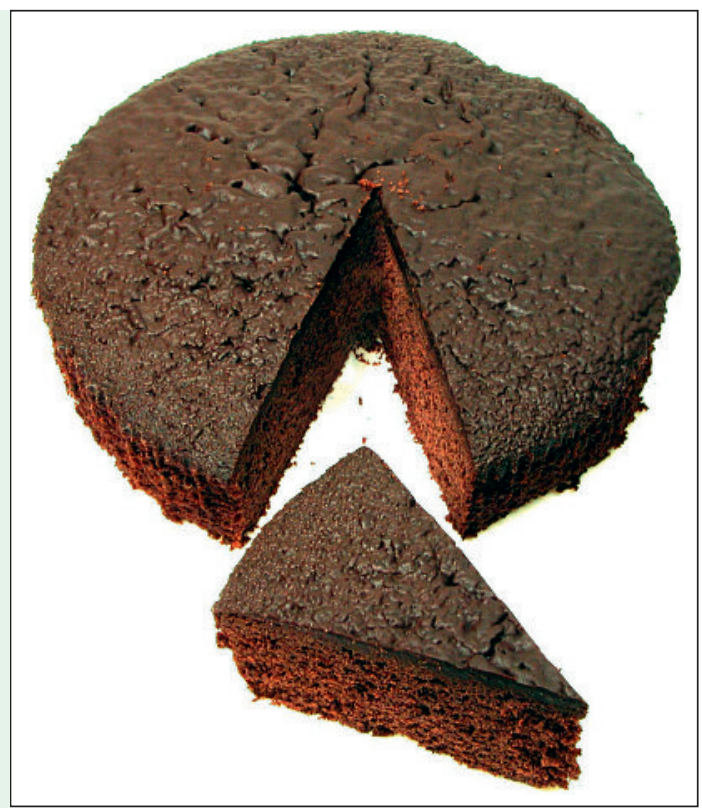

kriterien werden bei der Kostengutsprache im Voraus festgelegt. Nach dieser Testzeit muss das Ansprechen durch den Onkologen beurteilt werden. Lässt sich beim Patienten die Wirksamkeit auch im konkreten Einzelfall nachweisen, erfolgt eine zweite (zeitlich ebenfalls begrenzte) Kostengutsprache.

Eine besondere Regelung verlangt der adjuvante Einsatz (noch) nicht zugelassener Medikamente, weil die Wirksamkeit am Einzelfall nicht geprüft werden kann. Die initiale unentgeltliche Abgabe der Medikamente für einen bis zwei Monate ist kein taugliches Mittel, da adjuvante Therapien sich über Jahre hinziehen können. Stützt man sich auf den BGE, so soll durch den Off-label-use das Listensystem nicht ausgehöhlt werden. Dieser Forderung kann nachgekommen werden, wenn der Medikamentenpreis so gesenkt wird, dass die adjuvante Behandlung nicht mehr Kosten verursacht als die Behandlung mit dem gleichen Medikament in der palliativen Situation. Mit einer solchen Lösung kann die pharmazeutische Industrie dem Vorwurf begegnen, sie wolle die Registrierung umgehen und über den Off-label-use den Marktanteil ohne Preissenkung ausweiten.

Nach interner Diskussion in der Arbeitsgruppe Offlabel-use wurde ein Arbeitspapier* verabschiedet, das diese Punkte nun umzusetzen versucht, um so eine einheitliche Behandlung der Gesuche zu erreichen. Möglicherweise werden Krankenkassen im Off-label-Bereich in Zukunft gemeinsam Preisverhandlungen mit der Industrie führen, um bessere Konditionen zu erhalten und den administrativen Aufwand zu verkleinern. 